\title{
Identification d'une nouvelle protéine membranaire caractéristique des lymphocytes cytotoxiques humains in vivo
}

Lors d'une réponse immune vis-à-vis d'un agent pathogène ou d'une cellule tumorale, le système immunitaire stimule, au sein de la lignée lymphoïde T, l'expansion de lymphocytes effecteurs cytotoxiques. La particularité intrinsèque de ces cellules se manifeste par leur capacité à détruire spécifiquement et directement leurs cibles cellulaires. La caractéristique cytologique de ces lymphocytes est la présence de granules cytoplasmiques qui contiennent un arsenal d'enzymes protéolytiques qui se déversent dans le compartiment extracytoplasmique lors du contact cellulaire spécifique avec les cellules cibles. Ces dernières peuvent être des cellules allogéniques introduites lors d'une transplantation d'organe, des cellules tumorales ou bien des cellules infectées par un agent pathogène. De façon générale, dans le sang circulant d'un individu normal, il existe deux types de lymphocytes cytotoxiques, les lymphocytes tueurs naturels (NK, natural killer) qui sont les plus importants en nombre et les lymphocytes $\mathrm{T}$ cytotoxiques (CTL, otoloxic T lymphocyle). Contrairement aux lymphocytes $\mathrm{T}$ qui recomnaissent les cellules cibles par l'intermédiaire d'un récepteur protéique spécifique qui est le produit de gènes réarrangés (TCR, T cell receptor) constitué soit d'un hétérodimère $\alpha \beta[1]$, soit d'un hétérodimère $\gamma \delta[2]$, les lymphocytes NK n'expriment pas un tel récepteur.

En fait, bien que la structure membranaire impliquée dans la réactivité spécifique des lymphocytes NK n'ait pas été identifiée, nous savons de façon précise que cette dernière n'est pas le produit de segments de gènes qui subissent des réarrange$m / s n^{\circ} 12$ vol. 9, déremlime 93 ments somatiques au cours de la maturation lymphocytaire. Par ailleurs, la spécificité des cellules NK n'est pas clairement établie [3], alors que nous savons depuis plusieurs années que les CTL qui expriment un TCR $\alpha \beta$ réagissent avec des peptides antigéniques présentés principalement par les molécules de classe l du complexe majeur d'histocompatibilité (CMH). Quant aux CTL humains qui expriment un TCR $\gamma \delta$, là encore nous ne disposons que d'informations confuses sur leur(s) spécificité(s) [4]. Les lymphocytes NK expriment des glycoprotéines membranaires différentes de celles exprimées par les CTL, ils portent notamment des récepteurs de faible affinité pour les complexes immuns (CD56 = Fc $\gamma$ RIII) et les molécules CD56 (NCAM, neural cell adhesion molecule). (Ces molécules ne sont pas spécifiques des lymphocytes $\mathrm{NK}$ puisqu'elles sont portées par d'autres lignées cellulaires [5]. Les lymphocytes NK n'expriment pas les antigènes de différenciation (CD4 et n'expriment que très faiblement, pour une minorité d'entre eux, les antigènes de différenciation CD8. Bref, comme il n'existait pas de glycoprotéine membranaire spécifique comnue des lymphocytes cytotoxiques humains, nous avons néammoins fait l'hypothèse qu'une telle structure devait exister et nous nous sommes efforcés de la détecter. Certes, il existe des enzymes intracytoplasmiques, telle la "perforine" [6], qui sont caractéristiques de la fonction cytotoxique à médiation lymphocytaire, mais elles ne peuvent être utilisées comme marqueur potentiel pour isoler les cellules cytotoxiques dans le but d'une manipulation ex vivo à visée thérapeutique puisque la mise en évidence de ces enzymes nécessite la destruction des cellules. L'anticorps monoclonal représente l'outil le plus performant pour identifier des molécules membranaires et pour isoler les populations cellulaires qui les portent. C'est pourquoi nous avons développé depuis quelques années une stratégie de recherche qui consiste à produire des anticorps monoclonaux murins afin de découvrir des glycoprotéines membranaires lymphocytaires humaines caractéristiques d'une souspopulation fonctionnelle [7]. Pour isoler un anticorps monoclonal recomnaissant une molécule membranaire spécifique des lymphocytes cytotoxiques, nous avons immunisé des souris avec une lignée cellulaire humaine ayant une activité cytotoxique, puis nous avons prélevé les cellules spléniques de ces souris pour les fusionner avec un myélome murin suivant une procédure classique, dans le but de produire des hybridomes immortalisés sécréteurs d'anticorps.

Nous avons produit un anticorps monoclonal, que nous avons appelé BY55 [8], capable de réagir exclusivement avec les lymphocytes cytotoxiques humains. Ia structure reconnue par l'anticorps BY55 est une glycoprotéine d'un poids moléculaire de $80 \mathrm{kDa}$ qui est portée par les lymphocytes NK et CTI, $\alpha \beta$ et $\gamma \delta$ du sang circulant. Ce sont tous des lymphocytes à grains. L'utilisation de cet anticorps monoclonal a permis d'isoler à l'aide d'un trieur de cellules les lymphocytes humains cytotoxiques du sang circulant et de les cultiver $e x$ vituo pendant plusieurs semaines sans qu'ils perdent leur caractéristique fonctionnelle. Cee
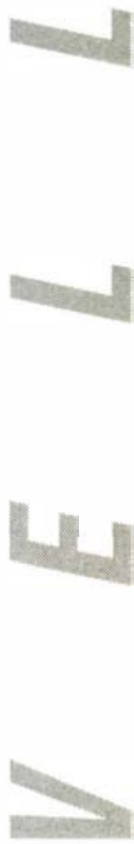
type de résultat nous conduit à penser que l'anticorps monoclonal BY55 pourrait représenter un outil extrêmement efficace dans les approches thérapeutiques, soit en cancérologie (manipulation et propagation ex vivo des cellules cytotoxiques), soit en transplantation de moelle osseuse (élimination des cellules cytotoxiques du greffon, responsables de la réaction du greffon contre l'hôte (GVH, graft versus host). Il est à noter que l'antigène BY55 est modulé négativement au cours de la mise en culture des lymphocytes cytotoxiques sans modifier leur activité tueuse, ce qui nous fait penser que cette molécule ou cet épitope n'intervient pas directement dans la fonction cytotoxique. Par ailleurs, nous pensons que la mise en évidence de ce marqueur va nous permettre de suivre les effecteurs $\mathrm{CD}^{+}$ cytoxiques chez des individus infectés par le virus de l'immunodéficience humaine. En effet, nous venons de montrer que le pourcen- tage de lymphocytes qui portent l'antigène BY55 est augmenté chez les individus asymptomatiques HIV positifs [9].

1. Bensussan A, David V. Le récepteur spécifique du lymphocyte $\Gamma$. méderine/sriences $1986 ; 2$ : 296-7.

2. Moingeon $P$, Jitsukawa $S$, Faure F, Troalen $F$, Triebel F, Graziani M, Forestier F, Bellet D, Bohuon C, Hercend T. A Ychain complex forms a functional receptor on cloned human lymphocytes with natural killer-like activity. Nature $1987 ; 325: 723-5$.

3. Christiansen FT, W'itt CS, Ciccone E, Townend D, Pende D, Viale D, Abraham IJ, Dawkins RI., Moretta L.. Human natural killer alloreactivity and its association with the major histocompatibility complex : ancestral haplotypes encode particular NK-defined haplotypes. J Exp Med 1993; 178: 1(133-9.
4. Bensussan A, David V, Vilmer E, Leca G, Boumsell L. Immunodeficiency after bone marrow transplantation can be associated with autoreactive TCR $\gamma \delta$-bearing lymphocytes. Immunol Rev 1990; 116: 5-13.

A.B. 5. Rochet N, Anderson P, Vivier E. Structure L.B. et fonction du complexe CD16: $\zeta: \gamma$ des cellules NK. médecine/sciences 1992; 8: 359-65.

6. Tschopp J, Nabholz M. Perforin-mediated target cell lysis by cytotoxic $T$ lymphocytes. Annu Rev Immunol 1990; 8: 279-85.

7. David V, Bachelez H, Leca G, Degos I. Boumsell L, Bensussan A. Identification of a novel $110 \mathrm{kDa}$ structure expressed on a subset of $\mathrm{TcR} \gamma \delta$ bearing cloned lymphocytes. / Immunol 199(); $114: 1-6$.

8. Maïza H, Leca G, Mansur IG, Schiavon V Boumsell I., Bensussan A. A novel 80kD cell surface structure identifies human circulating lymphocytes with natural killer activity. J Exp Med $1993 ; 178: 1121-6$.

9. Bensussan A, Rabian C, Schiavon V, Bengoufa D, Leca G, Boumsell L. Significant enlargement of a specific subset of CD3+C.D8+ peripheral blood leukocytes mediating cytotoxic T lymphocyte activity during human immunodeficiency virus infection. Proc Natl Acad Sci USA 1993; 90): 9427-30. dystrophine, par exemple, doit prendre de 12 à 18 heures pour être totalement transcrit in vivo, ce qui pourrait expliquer une partie de sa spécificité pour des myotubes, cellules post-mitotiques. À l'inverse, d'autres modèles établissent une certaine relation entre la réplication de l'ADN et la transcription. Selon ce schéma, la décondensation de la chromatine associée à la réplication de l'ADN serait un événement propice à la fixation sur ce dernier des différents éléments de la machinerie d'activation transcriptionnelle. Liu et al., du laboratoire de B. Alberts (CA, USA) [1], viennent de démontrer chez E. coli que la progression d'une fourche de réplication de l'ADN ne détachait pas l'ARN poly- mérase et ne déplaçait pas la chaîne naissante d'ARN en cours d'élongation. Ces résultats obtenus dans un système acellulaire in vitro mériteraient cependant d'être confirmés par des données in vivo, bien difficiles à obtenir. Néanmoins, ces données suggèrent que l'élongation des transcrits en cours de synthèse lors d'une division cellulaire peut reprendre dès la fin de celle-ci, constituant par conséquent une notable économie d'énergie pour les cellules qui n'ont pas à reprendre à zéro un travail de transcription qui eût été interrompu avant son terme par la réplication de l'ADN.

[Liu B, et al. Nature 1993; 366: 33-9.] 